Aus dem Institut fur Tierzucht und Haustiergenetik, Fachgebiet Tierhaltung und Haltungsbiologie, der Justus-LiebigUniversitat Gießen und der Hessischen Landesanstalt fur Tierzucht Neu-Ulrichstein

MEIKE SCHUDDEMAGE, STEFFEN HOY und KLAUS LANGE

\title{
Einfluß von Kunst- und Naturlicht auf das Verhalten beim Absamen und die spermatologischen Parameter von Rammlern
}

\author{
Herrn Professor Dr. Dr. H. H. Sambraus zum 65. Geburtstag gewidmet
}

\begin{abstract}
Summary
Title of the paper: Influence of artificial and natural light on behaviour during collection of semen and on spermatological parameters in rabbit bucks

39 bucks of New Zealand White hybrids were kept single over one year under three different light conditions (natural light - NL - with window - floor - relation of 1:20 and 27 Lux; artificial light - AL - with 8 or 16 hours light a day and a mean intensity of $60 \mathrm{Lux}$ ) and semen was collected once fortnight to analyse parameters of quality. At the same time sexual activity of bucks during collection of semen was assessed. Under NL conditions the highest sexual activity of male rabbits was found. But, decreasing length of day light in autumn led to a decrease in sexual activity. Best results for nearly all investigated semen parameters were detected in bucks kept under AL with 8 hours light a day.
\end{abstract}

Key Words: rabbit, buck, natural light, artificial light, semen quality, sexual activity

\section{Zusammenfassung}

39 Rammler (Weiße Neuseeländer Hybriden) wurden uber ein Jahr hinweg einzeln unter drei verschiedenen Lichtbedingungen gehalten: Naturlicht (NL) mit einem Fenster-Fußbodenverhältnis von $1: 20$ und einer mittleren Beleuchtungsstärke von 27 Lux sowie Kunstlicht (KL) mit 8 oder 16 Stunden Licht pro Tag und einer durchschnittlichen Lichtintensităt von 60 Lux. Die Tiere wurden einmal in 14 Tagen zur Analyse der Spermaqualităt abgesamt, wobei gleichzeitig das Verhalten beim Absamen beobachtet wurde. Unter Naturlicht zeichneten sich die männlichen Tiere durch die größte Sprungfreudigkeit aus. Mit abnehmender Lichttaglänge im Herbst verringerte sich allerdings die sexuelle Aktivităt. Rammler, die stăndig unter einem Kunstlichtprogramm mit 8 Stunden Licht pro Tag gehalten wurden, zeigten in nahezu allen spermatologischen Parametern die besten Ergebnisse.

Schlusselworter: Kaninchen, Rammler, Naturlicht, Kunstlicht, Spermaqualităt, sexuelle Aktivităt

\section{Einleitung}

Die Reproduktion beim Wildkaninchen (Oryctolagus cuniculus) unterliegt unter europäischen Bedingungen einem circannualen Zyklus (HAMMOND und MARSCHALL, 1925; BOYD, 1986), in dem die ersten Würfe im zeitigen Frühjahr fallen und im Spätsommer die Fortpflanzungssaison beendet wird. BRAMBELL (1944) und BOYD (1985) wiesen eine Atrophie der Hoden bei Wildkaninchen-Böcken mit abnehmender Lichttaglänge im Herbst/Winter nach. DAHLBÄCK und ANDERSSON (1981) sowie MEYER und STUBBE (1991) konnten einen Einfluß der Photoperiode auf die Ho- 
dengröße sowie saisonale Veränderungen der Leydigschen Zellen und der akzessorischen Geschlechtsdrüsen mit einem Maximum im Frühling und einem Abfall nach der Sommersonnenwende zeigen.

Weniger einheitlich sind die Ergebnisse zur möglichen Saisonalität der Fortpflanzung beim domestizierten Kaninchen. GRZIMEK (1979) und ELLENDORF (1990) gehen davon aus, daß - u.a. bedingt durch die ganzjährige Haltung im (teilklimatisierten) Stall und veränderte Fütterungs- und Ernährungsverhältnisse - eine saisonale Aktivität beim Hauskaninchen kaum noch vorhanden ist. Um dennoch einer möglichen verminderten Libido und Konzeptionsrate im Herbst und Winter zu begegnen, empfiehlt SCHLOLAUT (1998) eine gleichbleibende Beleuchtungsdauer von 14 bis 16 Stunden pro Tag. VIRÁG et al. (1992) konnten jedoch keine signifikanten Unterschiede im Ejakulationsvolumen, der Mobilität der Spermien sowie der Anzahl normaler Spermien in den Jahreszeiten feststellen, und KUTTNER (1974) sowie KUTTNER et al. (1975) wiesen darauf hin, daß eine entscheidende Rolle für die Spermaqualität der Absamrhythmus spielt, dem die Rammler unterzogen werden.

Die intensive Zucht von Kaninchen im Haupt- oder Nebenerwerb erfordert erhebliche Investitionen in Stallbau und Haltungstechnik, die sich über gleichbleibend hohe Leistungen bei niedrigen Verlusten während des Jahres amortisieren müssen. Die Anwendung von (Kunst-)Lichtprogrammen wird als eine Möglichkeit angesehen, mögliche saisonale Schwankungen zu überwinden und die Fruchtbarkeitsleistung zu stabilisieren und zu steigern. Die bisherigen Resultate zum Einfluß verschiedener Lichtprogramme auf die Spermaqualität der Böcke sind jedoch widersprüchlich.

UZCATEGUI und JOHNSTON (1990) fanden, daß kontinuierliche Lichtprogramme (14 Stunden Licht pro Tag) den intermittierenden Regimen (z.B. 2 × 8 Stunden Licht pro Tag) hinsichtlich der Spermaproduktion überlegen waren. Nach THEAUCLEMENT et al. (1995) besaßen Rammler unter täglich 16 Stunden Kunstlicht die besseren spermatologischen Eigenschaften als die Vergleichstiere mit 8 Stunden Licht, obwohl die sexuelle Aktivität der Tiere unter achtstündigem Lichttag höher als die bei einem 16stündigen Kunstlichtprogramm war. ORGEBIN-CHRIST (1968) meinte, daß sich der Aufenthalt von Kaninchenböcken unter konstanten photoperiodischen Bedingungen über längere Zeit ungünstig auf die Spermaproduktion auswirken würde. WALTER et al. (1968) wiederum ermittelten, daß die Hodenmasse von Rammlern bei 8 Stunden Licht pro Tag höher als bei 12- bzw. 16stündiger täglicher Beleuchtung war, woraus sie folgerten, daß ein gleichbleibend 8stündiger Lichttag die optimale Tageslichtlänge für Rammler sei.

Den eigenen Untersuchungen war somit das Ziel gesetzt, den Einfluß von Naturlicht im Vergleich zu zwei Kunstlichtregimen mit 8 bzw. 16 Stunden Lichttaglänge - über ein Jahr hinweg angewendet - auf das Verhalten der Rammler beim Absamen und auf die spermatologischen Parameter zu analysieren sowie darauf aufbauend Empfehlungen für die Anwendung von Lichtprogrammen in der Kaninchenzucht abzuleiten.

2. Material und Methoden

Die Untersuchungen fanden von Januar bis Dezember 1998 in der Hessischen Landesanstalt für Tierzucht Neu-Ulrichstein statt. Für die Untersuchungen standen drei wär- 
megedämmte und zwangsbelüftete Stallabteile (mittlere Stalltemperaturen im Untersuchungszeitraum zwischen 18,0 und $19,0^{\circ} \mathrm{C}$ ) zur Verfügung.

Es wurden drei verschiedene Lichtprogramme zeitlich synchron untersucht: Zwei Ställe mit Kunstlicht-Programmen (alle Fensterflächen lichtundurchlässig abgedunkelt) und ein Stall mit natürlichem Lichteinfall. Das Naturlichtregime (NL) war charakterisiert durch die Variation der Tageslichtlänge im Verlauf eines Jahres. Die Fensterfläche entsprach 1/20 der Bodenfläche des Stalles. Bei gleichmäßig bedecktem Himmel wurde hier eine mittlere Lichtintensität von 27 Lux ermittelt (Ein-EbenenMessung). Bei den Stallarbeiten gab es keine zusätzliche Beleuchtung. Die beiden Kunstlichtregime (KL) zeichneten sich durch eine Lichttaglänge von 8 Stunden (KL 8 , 7.00 bis $15.00 \mathrm{Uhr}$ ) bzw. 16 Stunden (KL 16, 4.00 bis $20.00 \mathrm{Uhr}$ ) über den gesamten Untersuchungszeitraum aus. Glühlampen mit einem Installationswert von $7,3 \mathrm{Watt} / \mathrm{m}^{2}$ wurden verwendet, was zu einer durchschnittlichen Lichtintensität von 60 Lux (gemessen in Ein-Ebenen-Messung) in beiden Kunstlichtstăllen führte.

Die für die Untersuchungen verwendeten Kaninchen (Weiße Neuseeländer Hybriden) entstammten dem Zuchtstamm der Hessischen Landesanstalt für Tierzucht. Für die Erfassung der Daten standen insgesamt 39 Rammler zur Verfügung $(N L=14$, KL $16=12$ und KL $8=13$ Tiere).

Die Rammler wurden jeweils in Einzelhaltung in serienmäßig gefertigten FlatdeckKäfigen der Größe $50 \mathrm{~cm}$ x $60 \mathrm{~cm}$ x $45 \mathrm{~cm}$ (Breite x Tiefe x Höhe) gehalten. Der Kunststoff-Rostenboden besaß eine Auftrittsbreite der Stege von $3 \mathrm{~cm}$ und eine Spaltenbreite von $1,3 \mathrm{~cm}$. Die Rückwand und die Seitenwände waren aus Aluminium, der vordere Teil des Käfigs und die obere Abdeckung bestanden aus Drahtgitter.

Alle Käfige waren mit einer Nippeltränke und einem Futterautomat ausgestattet. Die Kaninchen wurden mit einem pelletierten Alleinfuttermittel für Kaninchen (Alleinfuttermittel für trächtige und săugende Häsinnen sowie für Rammler und Masttiere) mit folgenden Inhaltsstoffen gefuittert:

Nährstoffe:

Rohprotein

Rohfaser

Rohfett

Rohasche

Lysin

Methionin/Cystin

Kalzium

Phosphor

Natrium
Zusatzstoffe je kg:

$\begin{array}{rlr}18 \% & \text { Zusatzstoffe je kg: } & 10000 \mathrm{IE} \\ 15 \% & \text { Vit. A } & 1200 \mathrm{IE} \\ 3 \% & \text { Vit. } \mathrm{D}_{3} & 50 \mathrm{mg} \\ 9 \% & \text { Vit. E } & 20 \mathrm{mg} \\ 0,8 \% & \text { Kupfer } & \\ 0,9 \% & & \\ 1 \% & & \\ 0,6 \% & & \\ 0,15 \% & & \end{array}$

Nach einer Empfehlung von SCHLOLAUT (1998) wurde zur Reduzierung des Fettansatzes nach dem Beginn der Geschlechtsreife der Tiere die Nährstoffzufuhr der Zuchttiere begrenzt. Den Rammlern wurde der Zugang zum Futter pro Tag auf fünf bis sechs Stunden beschränkt, um eine gute Zuchtkondition zu gewährleisten. Aus diätetischen Gründen bekamen alle Tiere regelmäßig etwas Heu. Wasser konnte von den Kaninchen ad libitum aufgenommen werden.

Alle Rammler befanden sich bis zum Beginn der Untersuchung unter dem Einfluß einer kontinuierlichen Lichttaglänge von gleichbleibend 14 Stunden. Die Verteilung der 
Tiere auf die verschiedenen Lichtprogramme erfolgte paritätisch nach der Lebendmasse zum Zeitpunkt des Untersuchungsbeginns.

Die Rammler wurden in einem 14tägigen Rhythmus abgesamt. Vor Untersuchungsbeginn wurden alle Rammler an ein Phantom und die künstliche Vagina gewöhnt. Bei Untersuchungsbeginn betrug das Alter der Rammler 154 Tage.

Die Spermagewinnung erfolgte mit Hilfe eines Phantoms und einer auf $45^{\circ} \mathrm{C}$ vorgewärmten künstlichen Vagina. Zur Bewertung der Sprungfreudigkeit wurde ein modifiziertes Schema nach BROCKHAUSEN et al. (1979) herangezogen. Die Bewertungskriterien waren: Spontane Annahme des Phantoms und der künstlichen Vagina ohne Zusetzen einer Häsin, Deckakt nur nach Zusetzen einer Häsin möglich und kein Deckakt auslösbar. Die Ejakulate wurden unmittelbar nach ihrer Entnahme untersucht. Zuerst wurden Volumen, Konsistenz, Farbe und das Vorhandensein von Beimengungen festgehalten. Ein vorhandener Schleimpfropf wurde sofort mit einer Pinzette entfernt, da er die Spermaqualität negativ beeinflußt (BUSCH et al., 1991). Anschließend wurden pH-Wert, Massenaktivität, Vorwärtsbewegung der Spermien, Anzahl der Spermien pro Milliliter und pro Ejakulat und der Anteil der morphologisch veränderten Spermien bestimmt. Ejakulate mit Urinbeimengung wurden von allen weitergehenden Untersuchungen ausgeschlossen.

Das Volumen der Spermaprobe wurde mit Hilfe graduierter Reagenzgläschen ermittelt. Die Spermafarbe wurde nach einem Schema klassifiziert (elfenbeinfarben, undurchsichtig, durchsichtig, gelblich). Die Konsistenz des Spermas wurde in vier graduelle Gruppen eingeteilt (rahm-ähnlich, milch-ähnlich, molke-ähnlich, wäßrig). Der pH-Wert wurde mittels Spezialindikatorpapier (Meßbereich 6,4 bis 8,0) gemessen. Zur Bestimmung der Massenaktivität wurde ein Tropfen des Ejakulates auf vorgewärmte Objektträger $\left(37^{\circ} \mathrm{C}\right)$ aufgetragen und bei Lupenvergrößerung unter einem Mikroskop mit Heiztisch beurteilt. Der Grad der Massenaktivität wurde nach einem Schema von 0 bis 5 nach WEITZE und MÜLLER (1991) eingestuft. Zur Beurteilung der Vorwärtsbewegung wurde dieser Tropfen mit einem vorgewärmten Deckglas abgedeckt und bei 400 facher Vergrößerung betrachtet. Die Vorwärtsbewegung wurde geschätzt und in Prozent angegeben. Zur Bestimmung der Anzahl der Spermien wurden die Spermaproben in einer Blutmischpipette für Erythrozyten mit 10 \%iger Natriumchloridlösung in einem Verhältnis 1:100 verdünnt und die Spermien in einer Zählkammer nach Thoma ausgezählt. Es wurden fünf Quadrate à 16 Kleinstquadrate gezählt und die Anzahl der Spermien pro Milliliter nach folgender Formel berechnet:

$$
\mathrm{n}=4000000 \times \mathrm{Z} \times \mathrm{V}
$$

$$
\begin{array}{ll}
\mathrm{n} & =\text { Anzahl der Spermien pro Milliliter } \\
4000000 & =\text { Volumen eines Kleinstquadrates }(1 / 4000000 \mathrm{ml}) \\
\mathrm{Z} & =\text { Zahl der pro Kleinstquadrat gezählten Spermien } \\
\mathrm{V} & =\text { Verdünnung }
\end{array}
$$

Die Anzahl der Spermien pro Ejakulat wurde aus der ausgezählten Anzahl der Spermien pro Milliliter und dem Ejakulationsvolumen berechnet. Zur Bestimmung des Anteils morphologisch veränderter Spermien wurden Ausstriche auf entfetteten Objektträgern angefertigt. Die Färbung erfolgte mit Anilin-Blau und Kristallviolett. Bei 1000 facher Vergrößerung (Öl-Immersion) wurden je Ausstrich 200 Spermien ausge- 
zählt und der Anteil der morphologisch veränderten Spermien nach BRETTSCHNEIDER (1948) ermittelt.

In der Zusammenfassung aller Absamungen konnte an 827 Fällen das Sprungverhalten der Rammler geprüft werden (282 NL, $285 \mathrm{KL} 16 \mathrm{~h}$ und $260 \mathrm{KL} 8 \mathrm{~h}$ ). 750 Ejakulate wurden auf Quantität und Qualität untersucht (261 NL, $260 \mathrm{KL} 16 \mathrm{~h}$ und $229 \mathrm{KL} 8 \mathrm{~h}$ ). An jedem Untersuchungstag wurde die Lebendmasse der Rammler erfaßt. Zusätzlich wurden die Länge und die Breite der Hoden mit Hilfe einer Schublehre gemessen. Aus den zwei gemessenen Größen wurde das Hodenvolumen errechnet. Als Modell diente ein Rotationsellipsoid mit folgender Formel:

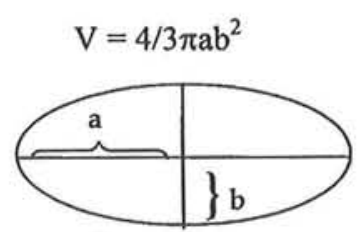

Um die Methode der Messung der Hodengröße intra vitam zu überprüfen, wurden die Tiere nach Beendigung der Untersuchungen geschlachtet. Es wurde die Hodengröße intra vitam kurz vor der Schlachtung und die Hodengröße unmittelbar post mortem an den präparierten Organen gemessen. Für den Zusammenhang zwischen Hodengröße intra vitam und der Hodengröße, die post mortem an den entnommenen Organen gemessen wurde, ergab sich ein Korrelationskoeffizient von 0,91 mit einem Signifikanzniveau kleiner $1 \%$ (Tab. 1).

Tabelle 1

Statistische Maßzahlen und Korrelationskoeffizient $r$ fur intra vitam und post mortem gemessene Hodenvolumen (Coefficient of correlation - $\mathrm{r}$ - for intra vitam and post mortem measured volume of testes)

\begin{tabular}{cccccccc}
\hline $\mathrm{n}$ & $\begin{array}{c}\text { Hodenvolumen } \\
\text { intra vitam }\left(\mathrm{cm}^{3}\right)\end{array}$ & $\mathrm{s}$ & $\begin{array}{c}\text { Hodenvolumen } \\
\text { post mortem }\left(\mathrm{cm}^{3}\right)\end{array}$ & $\mathrm{s}$ & $\mathrm{r}$ & $\mathrm{p}$ \\
\hline 30 & 8,77 & 1,29 & 6,83 & 1,03 & 0,91 & $* * *$ \\
\hline
\end{tabular}

Die Daten wurden mittels Microsoft Excel $97^{\mathrm{TM}}$ zu einer Matrix zusammengefaßt und mit dem Statistik-Programm-Paket SPSS 8.0 von SPSS Inc. für Microsoft Windows ${ }^{\text {TM }}$ statistisch bearbeitet. Dazu wurden die folgenden Verfahren für das gesamte Datenmaterial und für sortierte Teilstichproben angewendet:

- deskriptive Statistik mit der Berechnung der statistischen Maßzahlen (n, $\bar{x}, \mathrm{~s}$, Minimum, Maximum)

- Mittelwertvergleiche mit dem multiplen Test nach Student-Newman-Keuls

- Häufigkeitsvergleiche mit Chi-Quadrat-Unabhängigkeitstest in Kontingenztafeln

- Korrelations-/Regressionsanalyse.

\section{Ergebnisse}

3.1. Verhalten der Rammler beim Absamen

Unter dem Einfluß des Naturlichtes wiesen die Rammler die größte sexuelle Aktivität auf. Das spontane Absamen mit Phantom ohne Anwesenheit einer Häsin erfolgte in 
$20 \%$ aller Fälle. Nur bei $2 \%$ der Versuche konnte kein Deckakt ausgelöst werden. Im Kunstlichtregime mit 8 Stunden Licht pro Tag war in $15 \%$ der Absamversuche die Spermagewinnung ohne Zusetzen einer Häsin möglich, in $5 \%$ der Fälle wurde der Deckakt auf dem Phantom verweigert.

Das 16stündige Kunstlichtprogramm zeigte mit nur $8 \%$ der Fälle einer spontanen Spermaabnahme ohne Präsenz der Häsin im Vergleich zu den anderen Beleuchtungsvarianten den niedrigsten Wert. In $2 \%$ der Versuche ließ sich kein Sperma gewinnen (Abb. 1). Die Unterschiede im Verhalten der Rammler beim Absamen waren statistisch zu sichern $(\mathrm{p}<0,01)$.

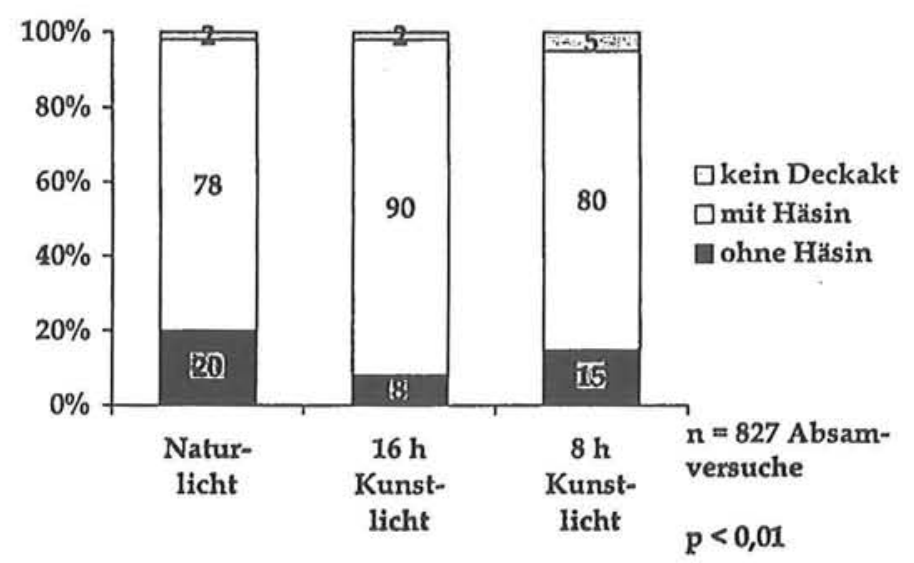

Abb. 1: Einfluß von Kunst- und Naturlicht auf das Verhalten der Rammler bei der Absamung (Influence of artificial and natural light on behaviour of bucks during semen collection)

\subsection{Spermatologische Parameter}

Die Rammler, die über ein Jahr hinweg kontinuierlich unter einem Kunstlichtregime mit 8 Stunden Licht pro Tag standen, besaßen in nahezu allen untersuchten Parametern (pH-Wert, Farbe, Konsistenz, Massenbewegung, Vorwärtsbewegung, Dichte, Volumen, Spermienzahl) die beste Spermaqualität (Tab. 2, Abb. 2). Nur bei der Häufigkeit

Tabelle 2

Einfluß von Kunst- und Naturlicht auf ausgewăhlte Spermamerkmale $(n=750$ Ejakulate) (Influence of artificial and natural light on selected semen parameters $-n=750$ ejaculates)

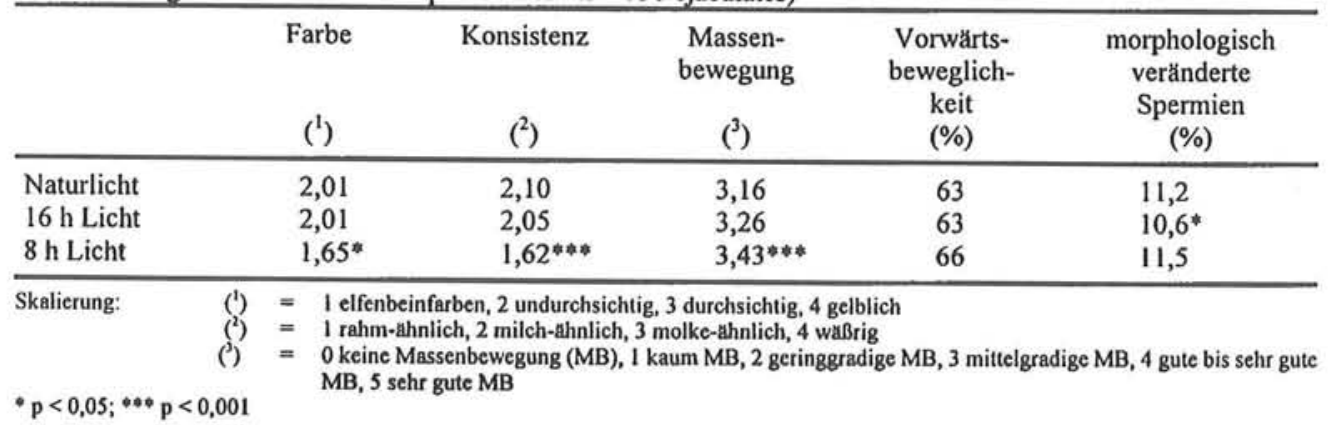


morphologisch veränderter Spermien trat bei den männlichen Tieren unter einem 16stündigen Kunstlichttag der niedrigste Wert (10,6 \%) auf. Bei den Rammlern unter dem Kurztag-Lichtregime (KL 8) war der Anteil veränderter Spermien um 0,9 \% höher als bei den Tieren unter einem Kunstlicht mit langer Beleuchtungsdauer (KL 16) und um $0,3 \%$ höher als bei den männlichen Tieren unter Naturlicht.

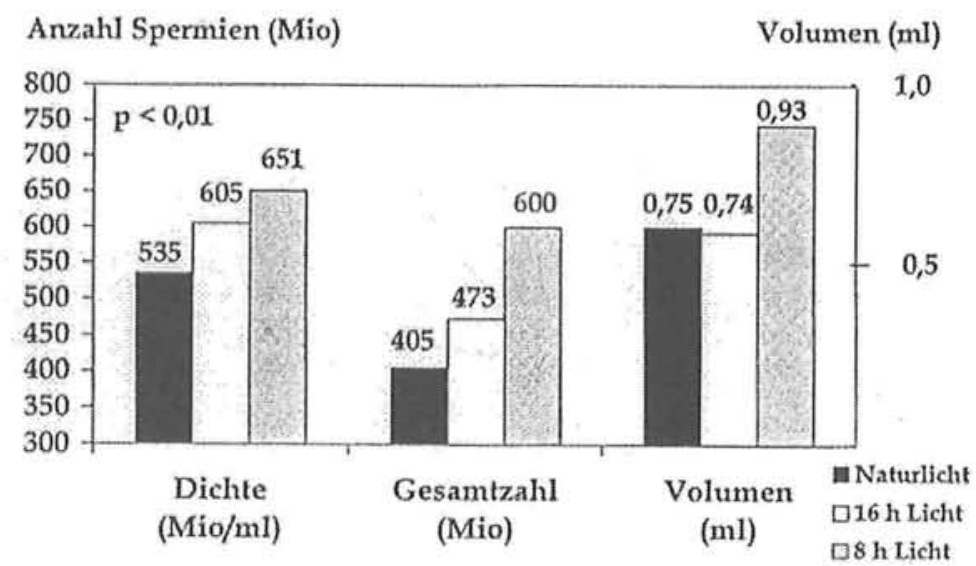

Abb. 2: Einfluß von Kunst- und Naturlicht auf Spermavolumen sowie Anzahl der Spermien pro Milliliter und pro Ejakulat ( $n=750$ untersuchte Spermaproben) (Influence of artificial and natural light on semen volume and number of sperms per milliliter and per ejaculate $-n=750$ analyzed semen probes)

Bei den Parametern Dichte des Spermas (Anzahl Spermien/ml), Gesamtzahl an Spermien im Ejakulat und Volumen erzielten die Rammler unter dem 8stündigen Kunstlichtprogramm die signifikant höchsten Werte (Abb. 2). Die Ejakulate der Tiere des Kurztagregimes (KL 8) hatten mit $651 \times 10^{6}$ Spermien pro Milliliter eine um 116 Mio Spermien/ml signifikant höhere Dichte als die der Kontrolltiere unter Naturlicht. Die Gesamtzahl der Spermien im Ejakulat betrug im kurzen Kunstlichttag 600 Mio Spermien, 195 Mio Spermien mehr als bei den Tieren unter Naturlichteinfluß. Das Ejakulat-Volumen erreichte im Kunstlichtprogramm KL 8 einen um nahezu 0,2 ml höheren Wert als bei den Vergleichstieren der beiden anderen Lichtregime (Abb. 2).

\subsection{Lebendmasse und Hodengröße}

Die mittlere Lebendmasse der Rammler in der Zusammenfassung aller Wägungen unterschied sich in den drei Beleuchtungsgruppen nur unwesentlich und nicht signifikant voneinander (Tab. 3).

Rammler unter dem Kunstlichtprogramm mit 16stündiger täglicher Beleuchtung besaßen in der Tendenz den höchsten Wert für die Hodengröße (Hodenvolumen) mit 8,79 $\mathrm{cm}^{3}$, die Tiere unter dem 8stündigen Kunstlichttag demgegenüber den geringsten Wert $\left(8,56 \mathrm{~cm}^{3}\right)$. Diese geringen Unterschiede ließen sich jedoch nicht statistisch sichern (Tab. 3).

Zwischen der Lebendmasse der Rammler und der Hodengröße intra vitam bestand 
Tabelle 3

Lebendmasse und Hodengröße intra vitam der Rammler unter verschiedenen Lichtbedingungen in der Zusammenfassung aller Messungen $(n=827, p>0,05)$ (Live weight and testes size intra vitam of bucks kept under different light conditions summarizing all measurements $-\mathrm{n}=827, \mathrm{p}>0,05$ )

\begin{tabular}{lccc}
\hline & $\begin{array}{c}\text { Anzahl } \\
\text { Tiere }\end{array}$ & $\begin{array}{c}\text { Lebendmasse }(\mathrm{kg}) \\
\bar{x} \pm \mathrm{s}\end{array}$ & $\begin{array}{c}\text { Hodengroße }\left(\mathrm{cm}^{3}\right) \\
\bar{x} \pm \mathrm{s}\end{array}$ \\
\hline Naturlicht & 14 & $4,3 \pm 0,38$ & $8,60 \pm 0,99$ \\
16 h Licht & 12 & $4,4 \pm 0,53$ & $8,79 \pm 1,31$ \\
8 h Licht & 13 & $4,3 \pm 0,39$ & $8,56 \pm 1,32$ \\
\hline
\end{tabular}

eine signifikante positive Korrelation mit einem Korrelationskoeffizient von $r=0,457$ und einem Bestimmtheitsmaß von 20,9\%. Der Zusammenhang zwischen Lebendmasse $(x)$ und Hodengröße $(y)$ kann durch die lineare Regressionsgleichung $y=1,47 x$ $+2,38$ beschrieben werden.

Bei der Prüfung der Zusammenhänge zwischen Hodengröße und spermatologischen Parametern konnte lediglich eine statistisch gesicherte positive Beziehung zwischen der Größe (dem Volumen) der Hoden und dem Ejakulationsvolumen $(r=0,344)$ mit einem Bestimmtheitsmaß von 11,8\% gefunden werden.

\subsection{Dynamik des Absamverhaltens und der Spermaqualität im Jahresverlauf}

Unter dem Einfluß des Naturlichtes war ein wachsender Anteil spontaner Absamungen ohne Anwesenheit einer Häsin von April (59 \%) bis Mai (Spitzenwert von $76 \%$ ) zu beobachten, nachdem die Werte im Januar und Februar noch zwischen 10 und $20 \%$ gelegen hatten. Im weiteren Verlauf des Jahres fiel die Quote spontaner Sprünge ohne Präsenz einer Häsin wieder auf Werte um $10 \% \mathrm{ab}$. In den beiden Kunstlichtregimen zeigte sich keine deutliche Dynamik im Absamverhalten über das Jahr hinweg.

Bei der Spermaqualität war lediglich ein markanter Abfall des Anteils morphologisch veränderter Spermien von etwa $20 \%$ bei den Jungrammlern zu Beginn der Untersuchungen auf ca. $10 \%$ bei Untersuchungsende auffallig - in allen drei LichtregimeGruppen annähernd gleich. Die detaillierte Ergebnisdarstellung kann der Arbeit von SCHÜDDEMAGE (2000) entnommen werden.

\section{Diskussion}

Kaninchen sind dämmerungs- und nachtaktive Tiere. Eine lange Dunkelphase scheint sich günstig auf die Spermaqualität der Rammler auszuwirken. Unter natürlichen Beleuchtungsverhältnissen beginnt bei Wildkaninchen im zeitigen Frühjahr die Fortpflanzungssaison, die Hodengröße nimmt zu und die sexuelle Aktivität der Rammler steigt an (DAHLBÄCK und ANDERSSON 1981; MEYER und STUBBE 1991). Auch in den eigenen Untersuchungen zeigten die Rammler im Naturlichtabteil in den Monaten April und Mai den höchsten Anteil spontaner Ejakulationen auf dem Phantom ohne Anwesenheit der Häsin (SCHÜDDEMAGE, 2000). Zugleich stieg in diesem Zeitraum auch die Hodengröße an. Danach konnte ein Absinken der Hodengröße in den Sommermonaten beobachtet werden (SCHÜDDEMAGE, 2000). Dies steht in Übereinstimmung mit den Ergebnissen von BRAMBELL (1944) und BOYD (1985). Die Hodengröße der Tiere aus den beiden Kunstlichtprogrammen zeigte dagegen keine signifikanten Veränderungen über den Untersuchungszeitraum hinweg. Dies be- 
Arch. Tierz. 43 (2000) 4

legt zugleich den Einfluß der saisonal sich verändernden Naturlichttaglänge auf den morphologischen und physiologischen Status der männlichen Gonaden (BRAMBELL, 1944; BOYD, 1985). Unter intensiven Zucht- und Haltungsbedingungen muß jedoch während des gesamten Jahres eine hohe Fortpflanzungsbereitschaft und -leistung der Vatertiere angestrebt werden. Insofern haben die Ergebnisse zu den günstigen Auswirkungen eines Kunstlichtregimes mit kurzer täglicher Beleuchtungsdauer von 8 Stunden auf sexuelle Aktivität und Spermaqualität praktische Relevanz. Die generell bessere Sprungfreudigkeit der Rammler unter einem Kurztag-Lichtregime (KL 8) gegenüber dem langen Lichttag (KL 16) wurde in ähnlicher Weise auch von THEAUCLÉMENT et al. (1995) beschrieben.

Mit Ausnahme des Anteils pathomorphologisch veränderter Spermien besaßen die unter einem 8stündigen Kunstlichttag gehaltenen männlichen Kaninchen die beste Spermaqualität. Gegenüber den anderen Parametern der Spermabeschaffenheit erscheint die bis zu 0,9\% höhere Quote veränderter Spermien bei den Tieren aus der KL 8-Gruppe aus physiologischer Sicht wenig bedeutsam. Nach SCHLOLAUT (1998) muß erst mit einem Anteil von mehr als $20 \%$ veränderter Spermien mit verminderter Fortpflanzungsfähigkeit gerechnet werden.

Die in den eigenen Untersuchungen ermittelten spermatologischen Parameter stimmen gut mit den im Schrifttum mitgeteilten Werten überein (Tab. 4). Der nachgewiesene Abfall der Frequenz morphologisch veränderter Spermien von $20 \%$ zu Beginn der Spermagewinnung bis auf etwa $10 \%$ nach einem Jahr Untersuchungsdauer in allen drei Licht-Gruppen steht sicher im Zusammenhang mit der regelmäßigen Absamung

Tabelle 4

Spermatologische Parameter von Kaninchenbð̋ken beim Vergleich der in den eigenen Untersuchungen festgestellten Werte mit den Literaturangaben (Spermatological parameters of bucks - results of own investigations and data of literature)

\begin{tabular}{|c|c|c|c|c|c|c|}
\hline Autor(en) & pH-Wert & $\begin{array}{l}\text { Volumen } \\
\text { (ml) }\end{array}$ & $\begin{array}{l}\text { Dichte } \\
(\mathrm{Mio} / \mathrm{ml})\end{array}$ & $\begin{array}{c}\text { Spermien } \\
\text { gesamt } \\
\text { (Mio) }\end{array}$ & $\begin{array}{l}\text { VWB } \\
(\%)\end{array}$ & $\begin{array}{c}\text { Anteil } \\
\text { abnormer } \\
\text { Spermien } \\
(\%)\end{array}$ \\
\hline $\begin{array}{l}\text { SCHÜLKE } \\
(1991)\end{array}$ & $6,6-7,5$ & 1,0 & $100-200$ & o.A. & o.A. & o.A. \\
\hline $\begin{array}{l}\text { BATTAGLANI et } \\
\text { al. (1992) }\end{array}$ & 7,3 & 0,76 & 561 & 420 & 76 & o.A. \\
\hline $\begin{array}{l}\text { FINZI et al. } \\
(1992)\end{array}$ & $7,3-7,7$ & $1,1-1,6$ & $206-250$ & $223-318$ & $71-78$ & o.A. \\
\hline $\begin{array}{l}\text { TACKE et al. } \\
\text { (1995) }\end{array}$ & 7,28 & 0,96 & 200 & o.A. & 67,2 & 21,2 \\
\hline $\begin{array}{l}\text { LOPEZ et al. } \\
\text { (1996) }\end{array}$ & o.A. & $0,6-0,65$ & $360-390$ & o.A. & o.A. & o.A. \\
\hline $\begin{array}{l}\text { DAL BOSCO et } \\
\text { al. (1996) }\end{array}$ & o.A. & 0,85 & 450 & o.A. & 77 & o.A. \\
\hline $\begin{array}{l}\text { Eigene Unter- } \\
\text { suchungen }\end{array}$ & $7,2-7,3$ & $0,74-0,93$ & $535-651$ & $405-600$ & $63-66$ & $10,6-11,5$ \\
\hline
\end{tabular}


und der körperlichen Entwicklung. Die Tiere waren zu Beginn der Spermagewinnung noch vergleichsweise jung. Auch der Anstieg der Gesamtzahl der Spermien während der ersten drei Untersuchungsmonate in allen Lichtregimen (SCHÜDDEMAGE, 2000) ist darauf zurückzuführen, daß die Tiere die sexuelle Reife erst im Verlauf der Untersuchung erreichten. Nach den Angaben von MAY und SIMPSON (1975) soll dies mit durchschnittlich 224 Tagen der Fall sein.

Beziehungen zwischen Hodengröße und spermatologischen Parametern, wie sie von CARSON und AMANN (1972) sowie SCHLOLAUT (1998) beschrieben wurden, lieBen sich nicht nachweisen. Lediglich ein signifikanter positiver Zusammenhang zwischen Hodengröße und Ejakulationsvolumen wurde gefunden $(r=0,344)$. In guter Übereinstimmung mit den Resultaten von THEAU-CLÉMENT et al. (1995) konnte eine Korrelation zwischen Lebendmasse und Hodenmasse der Rammler bestätigt werden. In der vorliegenden Analyse traten kaum Unterschiede in der Körpermasse zwischen den männlichen Tieren der drei Gruppen auf, so daß die Differenzen zwischen den spermatologischen Parametern nicht lebendmasseabhängig sein können. Die Ergebnisse der eigenen Untersuchungen gehen nicht konform mit französischen Resultaten (THEAU-CLÉMENT et al., 1995), wonach Rammler unter dem Einfluß einer Lichttaglänge von 16 Stunden den Tieren aus einem 8stündigen Kunstlichtprogramm in den Spermaqualitätsmerkmalen überlegen waren. Die quantitativen Spermaparameter waren allerdings nur im ersten Drittel der Untersuchungszeit bei den Tieren mit 16 Stunden täglicher Beleuchtung besser, ab der 15. Woche zeigten die Kaninchenböcke unter 8stündiger Lichttaglänge die günstigeren Ergebnisse. Die genannten Untersuchungen erstreckten sich nur über ein halbes Jahr, andere Versuchsanstellungen (z.B. ORGEBIN-CHRIST, 1968) waren noch kürzer (z.B. sieben Wochen). Somit war dieser Untersuchungszeitraum - angesichts des Jahresrhythmus des Fortpflanzungsgeschehens - offensichtlich zu kurz, um repräsentative Aussagen über ein geeignetes Lichtregime für Rammler zu entwickeln.

Nach den vorliegenden Untersuchungsergebnissen kann für Ställe ohne Fenster ein Kunstlichtprogramm mit 8 Stunden Licht pro Tag und einer Beleuchtungsstärke von 60 Lux (Ein-Ebenen-Messung) empfohlen werden. Dies entspricht auch den Anforderungen der Häsinnen an ein Kunstlichtregime zur Erzielung hoher und stabiler Fruchtbarkeitsergebnisse (SCHÜDDEMAGE, 2000). Es kann angenommen werden, daß sich aus der verbesserten Spermaqualität auch ein positiver Effekt hinsichtlich der Besamungsergebnisse ableiten läßt. Das war allerdings nicht Ziel der vorliegenden Untersuchungen und wurde nicht überprüft.

\section{Literatur}

BATTAGLANI, M.; CASTELLINI, C.; LATTAIOLI, P.: BOYD, I.L.:

Variability of the main characteristics of rabbit semen. J. Appl. Rabbit Res. 15 (1992), 439-446

Effect of photoperiod and melatonin on testis development and regression in Wild European Rabbits BOYD, I.L.: (Oryctolagus cuniculus). Biol. Reprod. 33 (1985), 21-29

BRAMBELL, F.W.R.:

The reproduction of wild rabbit Oryctolagus cuniculus. Proc. Zool. Soc. London 114 (1944), 1-45 
BRETTSCHNEIDER, L.H.:

Zit. in: BUSCH, W.; LOHLE, K.; PETER, W. (Hrsg.): Kunstliche Besamung bei Nutztieren. Gustav Fischer Verlag, Jena - Stuttgart 1991

BROCKHAUSEN, P.; PAUFLER, S.; SCHLOLAUT, W.:

Untersuchung zum Einfluß der Wärmebelastung durch das Wollvlies auf Spermaqualitătsparameter, Geschlechtsverhalten und Hodenvolumen beim Angorakaninchen. Zulchtungskunde, Stuttgart 51 (1979) 3, 234-248

BUSCH, W.; LÖHLE, K.; PETER, W.:

Künstliche Besamung bei Nutztieren. Gustav Fischer Verlag, Jena - Stuttgart 1991

CARSON, W.S.; AMANN, R.P.:

The male rabbit. VI. Effects of ejaculation and season on testicular size and function. J. Anim. Sci. 34 (1972) 2, 302-309

DAHLBÄCK, M.; ANDERSSON, M.:

Biology of the wild rabbit, Oryctolagus cuniculus, in southern Sweden. IV. Leydig cell activity and seasonal development of two male accessory organs of reproduction. Acta Zool. 62 (1981) 2, 113-120

DAL BOSCO, A.; SCUOTA, S.; CASTELLINI, C; CENCI, T.: Study of an artificial vagina to reduce the microbial contamination of rabbit semen. World Rabbit Science 4 (1996) 4, 201-204

ELLENDORF, F.:

Neuroendokrinologie induzierter Ovulation beim Kaninchen. Proc. 7. Arbeitstagung uber Haltung und Krankheiten der Kaninchen, Pelztiere und Heimtiere, Celle (1990), 35-39

FINZI, M.; MORERA, P.; KUZMINSKY, G.:

Effect of shearing on rabbit buck performance in hot ambient conditions. J. Appl. Rabbit Res. 15 (1992), 489-494

GRZIMEK, B.:

Grzimeks Tierleben, Enzyklopädie des Tierreichs. Deutscher Taschenbuch Verlag, München 1979

HAMMOND, J.; MARSHALL, F.H.A.:

Reproduction in the rabbit. Oliver and Boyd, Edinburgh 1925

KUTTNER, M.:

Die künstliche Besamung beim Kaninchen unter besonderer Berücksichtigung der Spermaverdünnung und -konservierung. Humboldt Univ. Berlin, Diss., 1974

KUTTNER, M.; LÖHLE, K.; SCHRAMM, R.:

Untersuchungen uber die künstliche Besamung beim Kaninchen unter besonderer Berücksichtigung der Spermaverdünnung und Spermakonservierung. Arch. Tierz., Berlin 18 (1975) 4, 247-254

LOPEZ, J.; ALVARINO, J.M.R.; DEL ARCO, J.A.; BUENO, A.; SANZ, C.:

Effect of male rabbit management on semen production. 6th World Rabbit Congress, Toulouse (1996), 83-86

MAY, D.; SIMPSON, K.B.:

Reproduction in the rabbit. Animal Breeding Abstracts 43 (1975) 6, 253-261

MEYER, R; STUBBE, M.:

Untersuchungen an einer Population des Wildkaninchens (Oryctolagus cuniculus; Linné, 1758) bei Weissenfels (Sachsen-Anhalt). Populationsőkologie von Kleinsăugearten (1991), 397-434

ORGEBIN-CHRIST, M.C.:

Gonadal and epididymal sperm reserves in the rabbit: Estimation of the daily sperm production. J. Reprod. Fert. 15 (1968), 15-25

SCHLOLAUT, W.:

Das große Buch vom Kaninchen. DLG-Verlag Frankfurt am Main 1998

SCHÜDDEMAGE, M.:

Untersuchungen zum Einfluß von Naturlicht im Vergleich zu zwei verschiedenen Kunstlichtregimen auf die Reproduktionsparameter weiblicher und männlicher Kaninchen (Oryctolagus cuniculus). Univ. Giessen, Diss., 2000

SCHÜLKE, B.:

Grundstruktur der Spermienzelle und Biochemie des Spermas. In: BUSCH, W.; LÖHLE, K.; PETER, W. (Hrsg.): Kunstliche Besamung bei Nutztieren. Gustav Fischer Verlag Jena - Stuttgart 1991

TACKE, S.; GÖTZE, S.; PINGEL, H.:

Einfluß der Häufigkeit der Spermagewinnung auf die Spermaqualităt der Rammler. Proc. 9. Arbeitstagung aber Haltung und Krankheiten der Kaninchen, Pelztiere und Heimtiere, Celle (1995), 4149 
THEAU-CLEMENT, M.; MICHEL, N.; ESPARBIE, J.; BOLET, G.:

Effects of artificial photoperiods on sexual behaviour and sperm output in the rabbit. Anim. Sci. 60 (1995), 143-149

UZCATEGUI, M.E.; JOHNSTON, N.P.:

Effect of continuous and intermittend photoperiods on the reproductive performance and growth of rabbits. J. Appl. Rabbit Res. 13 (1990), 215-219

VIRÁG, G.; MÉZES, M.; BERSÉNYI, A.:

Effect of independent factors on semen characteristics in rabbits. J. Appl. Rabbit Res. 15 (1992), 499-501

WALTER, M.-R.; MARTINET, L.; MORET, B.; THIBAULT, C.:

Régulation photopériodique de l'activité sexuelle chez le lapin mâle et femelle. Archives d'Anatomie d'Histologie et d'Embryologie 51 (1968), 773-780

WEITZE, K.F.; MÜLLER, E.:

Prinzipien der Spermauntersuchung. In: BUSCH, W.; LÖHLE, K.; PETER, W. (Hrsg.): Kunstliche Besamung bei Nutztieren. Gustav Fischer Verlag Jena - Stuttgart 1991

Eingegangen: 16.08.1999

Akzeptiert: 19.06 .2000

Anschriften der Verfasser

Dr. MEIKE SCHÜDDEMAGE, Prof. Dr. STEFFEN HOY

Justus-Liebig-Universitat Gießen, Institut furr Tierzucht und Haustiergenetik

Bismarckstraße 16

D-35390 Gießen

Dipl. Ing. agr. KLAUS LANGE

Hessische Landesanstalt fur Tierzucht Neu-Ulrichstein

D-35315 Homberg/Ohm 